\title{
Ten Key Steps to Writing a Protocol for a Qualitative Research Study: A Guide for Nurses and Health Professionals
}

\author{
Evridiki Kaba ${ }^{1}$, Areti Stavropoulou ${ }^{1}$, Martha Kelesi ${ }^{1}$, Aikaterini Triantafyllou ${ }^{2}$, Aspasia Goula ${ }^{3} \&$ Georgia Fasoi $^{1}$ \\ ${ }^{1}$ Department of Nursing, School of Health and Care Sciences, University of West Attica, Athens, Greece \\ ${ }^{2}$ School Nurse, Master of Science "Treatment and Care of Wounds and Ulcers”, Department of Nursing, School of \\ Health and Care Sciences, University of West Attica, Athens, Greece \\ ${ }^{3}$ Department of Business Administration, School of Administrative, Economics and Social Sciences, University \\ of West Attica, Athens, Greece
}

Correspondence: Evridiki Kaba, Department of Nursing, School of Health and Care Sciences, University of West Attica, Ag. Spyridonos Str., Egaleo 12243, Athens, Greece

\author{
Received: March 3, 2021 Accepted: April 24, 2021 Online Published: May 8, 2021 \\ doi:10.5539/gjhs.v13n6p58 URL: https://doi.org/10.5539/gjhs.v13n6p58
}

\begin{abstract}
Writing a well-structured research protocol is a critical component of any research activity. It is a demanding task that requires rigor and strenuous effort especially for the novice researchers in all disciplines. The aims of the present paper are a) to demonstrate the key steps required towriting a protocol for a qualitative research study b) to assist nurses and other health professionals in effectively developing protocols on qualitative research. For this purpose, an example qualitative research protocol was used entitled “Investigating nurses' views on care of mentally ill patients with skin injuries". This protocol was chosen because it provides a reasonable model of proposing a qualitative research design within the field of nursing. Results of this process led to the development of a 10 key-step guide to writing a protocol for a qualitative research study. A thorough analysis of how each step of the protocol must be undertaken and accomplished is presented and supported by the relevant literature. This paper provides an informative guide for novice researchers and/or nurse students, on how to develop successful protocols on qualitative research studies that guide research and decision making in naturalistic settings.
\end{abstract}

Keywords: research protocol, nursing students, qualitative research, grounded theory, nurses, health professionals

\section{Introduction}

A research protocol is necessary for the approval of every research project, whether it is submitted as a dissertation or thesis proposal or for research funding. Review of the relevant literature, revealed that the majority of the guidelines and examples given for writing a research protocol were mainly related to quantitative research. There were only few instructions for researchers who want to engage in qualitative research (Vivar et al., 2007)

The aims of the present paper are: a) to demonstrate the key steps required to writing a protocol for a qualitative research study b) to assist nurses and other health care professionals in effectively developing protocols on qualitative research studies.

Based on the literature (Drisko, 2005; Vivar et al., 2007; Stenious et al., 2017), a guide with 10 key-steps was designed and discussed. Research evidence that supported the reasoning of each step, was also included. In order to facilitate the development and the presentation of the 10 key-steps guide, an example research protocol, entitled “Investigating nurses' views on care of mentally ill patients with skin injuries", was used. This protocol was developed within the frame of a qualitative research study, using a Grounded Theory (GT) approach (Glaser \& Strauss, 1967).

The 10 key-steps included in the proposed research protocol guide, focus on the research topic and title formulation, on the background and rationale of the study, on the aims and objectives and on formulation of the research questions. Furthermore, the theoretical framework, and the methods of the proposed research are explored. Finally, data analysis, ethical considerations, limitations and strengths of the study and trustworthiness of the research are thoroughly discussed. This guide may help novice researchers, who want to conduct qualitative research, to develop a successful research protocol. 


\section{Step 1: Research Topic and Formulation of the Title}

The first and most important step of a research study is the formulation of the research topic, a problem that needs to be explored. This usually comes from a list of problems that nurses encounter in their daily practice. Once a research topic is chosen, a review of the literature needs to be conducted in order to explore what has already been studied in the field. Then the researcher will decide whether the research topic needs to be investigated or not. At this point he/she presents the background of the problem and recognizes any gaps in the literature in order to justify the significance of the study (Vivar et al., 2007). Having decided on the research topic, the researcher proceeds to form the title of the research.

Formulating a captivating and yet targeting title is one of the most important parts of the research protocol. When formulating the title of the research study it must be taken into account that this should clearly indicate the purpose of the research. The title need to be concise, informative, descriptive and specific and needs also to include keywords that are consistent with qualitative research such as: experiences, emotions, beliefs, knowledge, opinions (Bavdekar, 2016). The title of the example research protocol, which is used as a model to illustrate and support our rationale of the 10 key-steps process for writing a research protocol, was formed accordingly and included all the above mentioned notions. Relevant keywords were also included:

"Investigating nurses' views on care of mentally ill patients with skin injuries"

“Keywords: nurses' views, mentally ill patients, skin injuries, nursing care, qualitative research".

Bavdekar (2016), argues that the title should include some keywords, as this helps the article to be more likely accessed. The integration of all relevant keywords in the title ensures greater detectability and visibility through search engines. Generally a good title should:

- Inform the reader in a precise manner about the content of the article

- Be simple, direct, clear, compendious and appealing

- Avoid abbreviations or terminology

- Include keywords that facilitate the search

The title, which shows clearly the purpose of the study, will attract more readers who are really interested in this research field and possibly brings more citations to the article. The title is usually the first part of the article that someone reads and based on his/her understanding, the reader decides whether the article is interesting for him or not. Similarly, when readers search a database, usually read only the title of the papers. Therefore, the first impressions made by the title, make the readers to decide whether to read the paper or not. Generally it should contain 10-15 words. A long title may seem vague and boring. On the other hand, a short title may fail to inform readers about the content of the article (Bavdekar, 2016). As stated by Polit and Beck (2008), the title of a qualitative research study usually states the central phenomenon under study. According to Stenious et al., (2017) the authors need to decide the final version of the title only after the study is completed, in order to be sure that the title really captures the most accurate meaning of the study.

\section{Step 2: Background and Rationale}

At this step of the research protocol, information about the background of the study should be provided. The study should be placed in a broader social and scientific context: what is the background of the study and how the research will contribute to a further understanding of this situation. In addition, a justification for conducting the research should be highlighted. What is already known about the study topic and what and why new aspects need to be explored? The aim of this step is to illustrate and critically evaluate what has been researched so far in relation to the subject under study (Vivar et al., 2007). These aspects are clearly presented in the example protocol:

"Mentally ill patients are at high risk for physical illness with a significant lower survival rate (Hemingway, 2013). Mentally ill patients present an increased risk of injuries, such as pressure ulcers, diabetic ulcers, vascular ulcers, partial thickness burns, skin damages and wounds caused by self-injury (Pegram \& Bloomfield, 2010). However, mental health nurses mainly focus on the symptoms of mental illness, ignoring the cause of these injuries. Additionally, mentally ill patients have difficulty understanding the importance of wound management and the compliance with treatment. (Hemingway, 2013). The above factors, combined with the lack of nurses' special training on wound care, lead to complications and prolonged healing (Hinchliffe \& Linthwaite, 2019). Investigating nurses' views on the care of mentally ill patients with skin injuries could provide useful information and a better understanding of the problem, aiming to maximize the providing care". 
According to Hallberg (2010) in qualitative research the literature review is ambiguous and its purpose depends on the research approach of the study. In phenomenology, the researcher may conduct a literature review in order to determine if the area of interest as a lived experience has been researched or not, and also to identify new ideas. In historical research, doing a review of the literature is considered fundamental and crucial part of the research process. However, in grounded theory the researcher tries to avoid any review of the literature upon the beginning of the study, as he/she wants to avoid to "contaminate" the data with any preconceived ideas that might be considered as significant (Borbasi \& Jackson, 2012).

In conclusion, it is believed that in a qualitative research the review of the literature does not need to be an initial stage of the study and it would be good to be avoided at this stage. These views are grounded on the notion that qualitative research is based on the discovery of new ideas and the exploration of new fields. However, it is difficult to believe that there are still fields that have not been explored. For all the above reasons, the literature review in qualitative research should be brief and simply allow the researcher to become familiar with what has been published on the subject under study. At the same time, this process highlights the research gaps and demonstrates the significance of the proposed study (Flick, 2014).

\section{Step 3: Aims and Objectives}

The aim and the objectives of the intended research need to be clearly presented in the research protocol. Aims and objectives need to be clear, comprehensible and yet to demonstrate the nature of qualitative inquiry (Mantzoukas, 2007). In our example protocol, aims and objective of the study were formed as follows:

"The aim of this research study is to explore nurses'views on care of patients with mental illness with skin injuries. The objectives of the study are: a) To investigate the problems experiences by nurses who are dealing with the care of patients with skin injuries in the environment of a psychiatric hospital, b) to examine and identify the factors that influence nurses' views on the phenomenon under study".

The research problem can be stated as a research question or as an objective when the study aims to investigate a specific phenomenon (Mantzoukas, 2007).

\section{Step 4: Formulation of Research Questions}

At this stage, the researcher need to formulate the research questions. As qualitative research questions differ from quantitative research questions because they seek to explore or describe phenomena, and not to provide an explanation, they are often more general and more articulated. The researcher tries to understand the experiences, attitudes, and opinions that people have about the concepts in the research question (Houser, 2019). The research questions of our example research protocol follow:

- How psychiatric nurses experience the problems encountered while caring for mentally ill patients with skin injuries?

- What factors might affect their views and attitudes?

- What factors might influence the care delivered?

According to Issari and Pourkos (2015), in qualitative research, the research questions are broader than the hypotheses in quantitative research. They should be open, requiring a general direction and not to seek for particular answers. Their purpose is to define and explain all the unexpected characteristics of the phenomenon under study. Unlike quantitative research, the research questions in qualitative research, need to be flexible and able to be modified according to the researcher's judgement and decision during the research process.

As Matzoukas (2007) stated, the purpose of the qualitative research is to provide answers to "how" and "why". Therefore, the research question in a qualitative study must contain the above keywords. For example, it should begin with the following form: "understanding in depth the perceptions of... about how..." or "exploring the beliefs of... why..." or "interpreting the experiences of". The research question should not be treated with its strict grammatical term. It is not required to have a question style or to end in a question mark, because it is not intended to give a concrete answer to a predetermined question. Quite simply, this happens in qualitative research because the research questions are basically created during the process of data collection. As the researcher in not aware of the unique experiences and beliefs of the research participants cannot construct a predetermined research question for them. However, he/she will construct unique questions each time for each unique experience or belief of each participant. In fact, what constitutes the research question for qualitative research is the clear statement of a central approach on a phenomenon or issue. Thus, the research question on qualitative research methodologies takes the form of a clear statement on a subject. 


\section{Step 5: Theoretical Framework}

At this step, the researcher needs to justify why the proposed study design is appropriate to fill the gaps in the literature. The researcher need to explain why he/she chose to conduct a qualitative study and give details about the specific methodology employed (Connell, 1999).

According to Mantzoukas (2004), the researcher's goal in qualitative research is to investigate and get an in-depth understanding of participants' views, experiences and attitudes regarding a phenomenon. The qualitative researcher attempts to create a deeper, more complete and more elaborate understanding of the phenomenon under investigation, which will contain a holistic and deeper interpretation of the participants and their environment in relation to the research phenomenon.

In the example research protocol, it was clearly highlighted that GT was used to gain an in-depth understanding and interpretation of the phenomena being studied. At the same time, it was stressed that this was the ideal method for cases where an issue is being investigated for the first time, in order to better understand it. Furthermore, the researchers underscored that it is appropriate when little is known about a phenomenon and the purpose is to construct an explanatory theory (Glaser \& Strauss 1967). It is useful when research is dealing with areas where there are large gaps in understanding and where a new perspective is needed (Mantzoukas, 2004).

"Based on the principles of Grounded Theory, a qualitative approach will be used for the present study. The qualitative approach, is the most ideal approach for studying and investigating personal experiences, values and attitudes. Specifically, the qualitative approach has been described in the literature as the most appropriate methodology for assessing the views, attitudes and experiences of nurses (Gantley, 1999). Grounded Theory will be used to gain an in-depth understanding and interpretation of the phenomena under investigation. At the same time, it is an ideal method for cases where an issue is being investigated for the first time, in order to better understand it (Glaser \& Strauss 1967). In recent years, studies on the subject under study are really few, especially in our country, and for this reason it is considered appropriate to further and in-depth study this issue".

Connell (1999) argued that the researcher should always state the reasons why research is important and how it will contribute to the study of the phenomenon, why the study will be important and how it will add to the knowledge about the phenomenon under study. In the same vein, a clear and convincing statement of the qualitative approach used is of pivotal importance for a well-developed qualitative research protocol.

\section{Step 6: Methods}

This step should explain how the research study will be conducted. Details will be provided regarding the proposed sampling strategy (6.1), the potential participants' characteristics and recruitment (6.2), the data collection tool (6.3) and the process of data collection (6.4).

\subsection{Sampling Strategy}

Information should be given on how participants will be selected (convenience sampling, purposive sampling, and theoretical sampling). The choice of the specific sampling strategy used, should be justified, taking into account the specific qualitative research approach which will be applied. In our example protocol the sampling strategy was described along the lines of GT approach:

"A purposive sample will be used so that the participants to be typical cases and be able to provide in-depth and comprehensive answers on the subject under study. Nurses working in psychiatric wards with an increased frequency of skin injuries will consist the study population. Initially, 3 participants will be selected, as purposive sample, and then a theoretical sampling will be carried out as defined by the principles of Grounded Theory".

In qualitative research, specific participants are chosen because they have experienced the phenomenon under study. This kind of sampling can achieve a richness in the data and is known as purposive or purposeful sampling (Polit \& Beck, 2008). The aim of this sampling strategy is to select participants who will be able to provide the most relevant data for the research (Yin, 2011). According to Issari and Pourkos (2015), sampling in qualitative research, aims mainly, to identify what Patton (2002: p 230) calls information-rich cases, that "are offered for in depth study" and from which "one can learn a lot about issues of central importance for the purpose of research". Using a purposive sampling strategy (purposeful or purposive or judgmental sampling) means that the researcher actively and deliberately selects the sample whose members will best serve the aims and questions of his/her research. This sampling technique consists a form of non-probability sampling (Polit \& Beck, 2006). Charmaz (2014) defined as purposeful sampling, an a priori sample, used in most qualitative studies, and decided before the beginning of data collection process. In GT studies, theoretical sampling is used to identify evidence from the analysis, fill gaps, clarify doubts, and test explanations as the study progresses. Depending on the results from the 
first round of data analysis, the researcher may recruit more participants to interview, people who will confirm or disagree with what the researcher has already found. The researcher continues the theoretical sampling, moving back and forth between sampling, data collection, and data analysis, until data saturation is achieved, when there are no more information to be emerged (Bernard, 2002; Polit \& Beck, 2006).

Qualitative samples are often small (Fossey et al., 2002), but this is not usually a problem as the researcher is not attempting to generalize the findings. The aim of qualitative research is to gather rich information and not to estimate the percentage of the population that behaves or feels in some way. According to Mantzoukas (2007), in qualitative research the sample usually is a double-digit or even a single-digit number. A large sample can neither serve the aims nor the objectives of qualitative research, but instead works negatively for its validity, as in the case of a large number of participants the subjective and individualized characteristics are lost, which actually the qualitative research aim to understand.

Finally, selecting appropriate sample sizes in qualitative research has raised several debates and uncertainties among researchers. It is thus suggested qualitative researchers to strive towards justification of their sample size as this consist an important indication of the quality of qualitative research (Vasileiou et al., 2018).

\subsection{Participants' Characteristics and Recruitment}

In this section, the researcher may provide information on the potential participants' characteristics and the inclusion and exclusion criteria to the study. If so, the criteria for selecting and excluding the research participants should be explicitly defined and justified. Details must be also provided about the process followed for identifying the potential participants. Questions should be also answered such as how the participants will be identified and how they will be invited to participate. An illustration of our example research protocol is following:

Inclusion criteria will be:

- Nurses working in psychiatric wards, caring for mentally ill patients with a high frequency of skin injuries.

Exclusion criteria will be:

- Nurses working in psychiatric wards, caring for mentally ill patients with a small frequency of skin injuries or nurses with managerial roles, or part-time nurses, as they may have limited contact with patients.

The relevant literature stressed that in qualitative research, the researcher does not need to strictly identify the population under investigation, nor to strictly formulate the inclusion and exclusion criteria of participants, as it happens in quantitative research. This is because in qualitative research the sample can be adjusted according to the conditions and the design of the study and affected by the information that the researchers gradually get from the participants (Polit \& Beck, 2006). However, these information may assist especially the novice researcher to develop a comprehensive and realistic research plan.

\subsection{Data Collection Tool}

In data collection section, the researcher should present the specific tools and activities which will be used for the data collection phase. After having decided on the qualitative approach to be used, the next decision concerns the type of method (methods) that will be used for data collection. The most commonly used methods for collecting qualitative data are interviews, focus groups and observation. The choice of methods will depend on the aim of the study, the methodology and the type of information required. In addition, the available human and material resources need to be considered (Vivar et al., 2007). In our example research protocol, it was clearly stated by the researchers that interviews will be used as a data collection tool:

"Unstructured interviews will be used for data collection purposes. In particular, the unstructured interview is open and does not include predefined questions, but broad topics on which participants are asked to speak or express their opinions freely and on their own terms".

The types of interviews used in qualitative research, according on their degree of construction, can be distinguished in semi-structured and unstructured qualitative interviews (Issari and Pourkos (2015). These types of interviews help the researcher to collect as much data as possible by making the participants to talk freely and in depth about their experiences, perceptions and attitudes (Robson, 2010).

The semi-structured interview is often used by novice researchers, as it can work as a guide with a list of questions about topics that they want to cover in the interview. This particular type of interview is flexible as the content of the questions can be modified depending on the participant. The researcher can also be more flexible, e.g. to investigate some issues more exhaustively, to use the questions regardless their order, and to add or remove questions or topics (Issari \& Pourkos, 2015). 
The unstructured interview is open and does not include predefined questions, but rather the participant can reply freely on broad topics. Specifically, the researcher usually makes a list of 7 to 8 important topics which are used to develop an interaction with the participant (Robson, 2010, Mason, 2018).

Qualitative research designs hardly use structured interviews, as these are based on planned questions, and restrict the in depth collection of data or the development of new themes (Robson, 2010).

Qualitative interviews can be conducted one by one (individually), as well as within a group. Focus groups are a method of producing rich qualitative data through a process of interacting with team members. Focus groups concern with a group discussion in which participants are asked to talk to each other about a specific topic through an interaction process. Interaction between team members results in less influence from the researchers on the process and in giving more emphasis on participants' responses (Krueger, 1988; Frey \& Fontana, 1993; Wilkinson, 1998).

\subsection{Conducting the Interviews}

Following the recruitment process, the characteristics of the site for conducting the interviews, such as a private location inside or outside the hospital, and the type of material and equipment that will be needed should be described. Accordingly, in our example research protocol the following information was included:

'Before the commencement of the interview, the researcher will choose a convenient place and time for the participants, maintaining participants' privacy and data protection. The interviews will be tape-recorded and notes will be kept in order to capture all the clues from the non-verbal communication Before the interview, the researcher will give participants all the information about the study, the expected benefits of the research, issues of confidentiality and participants' rights. A consent form will be signed by each participant before the interview".

The main concern of the researcher is to determine the place and time of the interview. Specifying the right place and time so that the interview can be conducted smoothly, is of utmost importance for the data collection phase. The appropriate place is defined as a location that is quiet and easily accessible by the participant. In parallel, the best time for conducting an interview is the one that does not create stress or any other burden to the participants or the setting where the study is taking place (Kedraka, 2008).

The researcher should also provide information and justify his/her decision on recoding and transcribing the interview data. Tape recording and keeping notes are the most common procedure. According to Kedraka (2008), the reading of the material also depends on the way it is recorded. If the interview is recorded, it involves a time-consuming but necessary process of transcribing, which turns the verbal speech into written text, so it can be then systematically analyzed. It is suggested to combine the use of notes with recording, as notes may contain information that is not disclosed in the recorded text of the discussion (such as, non-verbal communication points, feelings before and after the interview).

According to Issari and Pourkos (2015), individual interviews or focus groups are usually recorded. However, there are some cases in which, due to the sensitivity of the issue or due to not consent for recording by the respondents, the interviewers need to keep detailed and clear written notes. The decision of how detailed the recording should be, is guided by the research questions and the method of analysis. However, it should be noted that the transcription is a kind of translating the participants' words, and only if it is combined with the written notes can be seen as a completed interview (Issari \& Pourkos, 2015; Willig, 2008).

The researcher should also provide details about the type of information that will be given to the potential participants before their involvement in the study. An informed consent form is included in the research protocol which will be provided to the study participants before the commencement of the interview.

Participants have the right to be informed about the aim of the study, the type of information being asked, how these information will be used and if any consequences may emerge due to their participation in the study. This moral concept is known as autonomy (Beauchamp \& Childress 2001).

Since qualitative data collection methods, such as interviews, focus groups and observation, are processes that require interaction between humans, possible risks may emerge, such as embarrassment, disagreements, and misconceptions (Rice \& Ezzy 1999). In qualitative interviews, the researcher encourages the participants to "open up" and explain how they experience the phenomenon. It is possible the participants to discuss personal information that they do not want to reveal, or to revive sad experiences related to the subject being studied. The researcher need to negotiate with the participants and determine if they feel comfortable to continue the interview or prefer to withdraw (Polit \& Beck, 2006).

The informed consent entails that the participants are given verbal and written information about the aim of the 
study and details about their participation. Participants should be eligible to ask for clarifications before signing the consent form. A copy of the signed form acts as prove of their agreement (Vivar et al., 2007)

\section{Step 7. Data Analysis}

At this step of the research protocol, the methods selected for data analysis should be described, reflecting the theoretical and methodological perspectives of the study. A description of how the data will be coded and developed into important categories and themes, from which participants' theoretical narratives can be drawn, should also be given. In the example research protocol the following information were included:

"“'The researcher, following the GT principles, will collect and analyze the data simultaneously, deciding whether he will collect more data. This is what is called constant comparative analysis. Collection of data will continue until no new or relevant ideas to be emerging from the collected data, a stage that is called 'theoretical saturation'. The constant comparative method and the theoretical sampling constitute the core of qualitative analysis in the grounded theory approach (Glaser and Strauss 1967). This process will be done by 'coding' the data,that is, forming categories, allocating codes for each word or phrase and shorting these codes into categories and themes. Then a core category will emerge and all the properties and relations between categories will be revealed."

According to Houser (2018), unlike quantitative studies, where analysis is postponed until all data have been collected, qualitative analysis begins almost as soon as data collection begins. During the collection, the data are examined and re-examined, and detailed memos are written. The recently collected data are compared with the existing data to confirm or refute the conclusions and to decide when saturation will be achieved. This continuous comparison of new results with existing results is a key feature of qualitative analysis. It is sometimes referred to as "intensive data engagement" to illustrate the depth at which the analyst examines the meaning of the information collected (Creswell \& Plano-Clark, 2011).

This approach allows the researcher to search for interesting ideas or to unravel complex information while the informants are still available and the data collection is in progress. Actually, the researcher is guided by the participants, instead of following a predetermined course that leads to an individual conclusion. In the case of GT -the qualitative research approach that used in our example research protocol-the continuous comparison of the data allows the researcher to guide the next data collection, select new participants or change the initial selection criteria to clarify issues that arise during the analysis. These secondary cases -known as theoretical sampling- are purposively selected to be compared with the sample already being studied. Although this may be perceived as a problem in sampling, theoretical sampling is, in fact, a source of triangulation, that is, it is one of the means by which qualitative analysts can confirm their results (Patton, 1999).

Although the analysis process is often done manually, it can also be done by using a computer software (Vaughn \& Turner 2016). The use of a computer-based qualitative analysis is controversial among qualitative researchers. Some believe that a process that is treated as holistic, interpretive, and humanitarian becomes mechanical with the use of automated systems. Others believe that automation through its efficiency will tempt qualitative researchers to use larger samples, thus sacrificing depth in order to be able to manage the range (King \& Horrocks, 2010). Some other researchers believe that using a computer for qualitative analysis makes the process faster and easier, while at the same time the researcher does not lose contact with the data. Through automated analysis, coding becomes less demanding, giving the researcher the opportunity to discover creative ways to deal with his data. By offering help in processing, storing, retrieving, registering, and sorting data, software for qualitative analysis leaves the researcher with more time to interpret and reflect the meaning. Such software allows transcribed data to be entered and encoded based on a code dictionary that will be developed by the researcher in an identical manner as that created by hand (Morris \& Ecclesfield, 2011).

However, there is a view that software cannot yet perform reflection, analysis, and interpretation. These tasks require the involvement of the human element, that is, a specialized qualitative researcher. Many qualitative researchers prefer manual analysis because they believe it allows them to stay immersed in the data despite the fact that it is a difficult and time consuming process. (Denzin \& Lincoln, 2011).

\section{Step 8. Ethical Considerations}

In this step, a statement on how the principles of research ethics will be followed and how they will be achieved and maintained during research, need to be mentioned. Also, the ethics committees that will approve the study, should be named. For example, the researcher should clearly state that before the commencement of the study, approval will be granted by the involved organization and the study participants and details on assuring ethics of the research will be provided:

"The researcher has to get an ethical approval by the Scientific Committee of the involved hospitals. The 
researcher needs to follow the Code of Nursing Ethics (articles 17, 18, 19) (Fowler, et al, 2010) and to ensure that participants will remain anonymous, their participation is voluntary and confidentiality of personal data will be kept. The researcher will inform participants about their right to withdraw from the study any time they might wish."

Ethics Committee's or Institutional Review Board's approval has to be sought before the study begins. The role of ethics committees and institutional review boards is to determine that the study is being adhered to all the ethical principles and that participants are protected from potential sources of harm (Burns \& Grove, 2001). Furthermore, in qualitative research it is important to ensure that participants' rights are protected. (Ryan et al., 2007). In addition to that, the researcher must anticipate any ethical issues that may arise during the research process (Creswell, 2009). Qualitative research involves collecting data from humans and for humans (Punch, 2005). Researchers need to protect research participants by building trust and protecting them from misconduct and any inappropriate actions (Creswell, 2009). First of all, the researcher has the obligation to respect the rights, needs, values and desires of the participants. Qualitative research attracts sensitive and in-depth answers to questions that make sense from statements and opinions (Sanjari et al., 2014)

\section{Step 9. Limitations and Strengths of the Proposed Study}

Researchers need to foresee potential limitations of the proposed study. For example accessibility problems, potential difficulties in recruitment as well as sensitive sample issues should be reported in the research protocol. Researchers need also to mention that despite the potential limitations, the findings of the study may offer an in-depth understanding of the phenomenon under investigation, and serve as valuable data to guide further studies.

Furthermore at this step of the qualitative research protocol, the expected benefits of the proposed research should be mentioned. Potential implication to practice should be also presented as these demonstrate the value of the proposed study. The reasons why the research is important to the scientific community need to be clarified, as well as the ways in which it can help providing knowledge. Example research protocol included relevant information:

"Accessibility in the proposed study setting might be a possible limitation of the study, as the health care services for individuals with mental problems are usually difficult to contact. In addition recruitment of potential participants might be a limitation as health professionals who work in such health care services are reluctant to participate in relevant research studies due to heavy work load and lack of time availability.

The strengths of the proposed study lay on the fact that studies on nurses'views regarding the care of mentally ill patients with skin injury are really few, especially in our country. Therefore this study generates new knowledge for the scientific community by exploring and understanding the views of nurses caring for this particular population. In addition, its contribution could be particularly important in improving the care provided to patients with skin damages, both in mental health services and in other health care services. At the same time, the results can be used to develop action plans and continuing education programs, either by nurses or by the hospital administration. Finally, the results of the research can provide the impetus for new studies on the subject under study, aiming at a more in-depth understanding".

This step requires time, commitment and reflection. Finally, at this point the researcher may point out the gaps in the literature and justify once again the need to conduct the proposed study (Vivar et al., 2007).

\section{Step 10. Trustworthiness of the Proposed Study}

As Ryan et al., (2007) state, the challenge in qualitative approaches is to produce reliable and credible evidence that demonstrate rigor. Rigor is the means of demonstrating the reliability, credibility and integrity of the qualitative research process. When the reader is able to audit the actions, influences and the events described by the researcher, then the rigor, or trustworthiness, of the study can be confirmed (Beauchamp \& Childress, 2001). The seminal work of Guba and Lincoln (1989) proposed the idea that qualitative research is based on trustworthiness rather than reliability and validity. The most common categories of quality criteria applied to assess the key elements of trustworthiness are credibility, dependability, transferability and confirmability According to Houser (2018), strategies to establish trustworthiness in qualitative research involve the prolonged engagement, persistent observation, researcher triangulation, audit trails, reflexivity, member check and thick descriptions. Qualitative researchers should refer to some of those issues in their research protocols. These issues are clearly demonstrated to our example research protocol:

"The researcher can enhance the credibility and establish the trustworthiness of the study, by having prolonged engagement, that is, investing sufficient time in the data collection activities in order to learn the culture of the group under investigation, For this reason the interviewer will be involved in the area of investigation long before the commencement of data collection; by visiting the mental health hospital, being introduced to nurses and 
talking to them".

\section{Conclusion}

This paper has provided a guide of the 10 key - steps necessary for the researchers who want to develop a protocol foa a qualitative research study. The reasoning of each step, examples of a given research protocol, and relevant literature that support each step were presented. This guide demonstrates that the development of a protocol for a qualitative research study is a systematic process that requires time, focus, knowledge, careful planning, as well as a comprehensive and a thorough approach to study design. It may also act as a 'manual' for the novice researchers to ensure adherence to the study methods, by monitoring the study's process and evaluating its outcomes. A sound research protocol yields a reliable research design and help the review bodies to fully understand the proposed study.

\section{Competing Interests Statement}

The authors declare that there are no competing or potential conflicts of interest.

\section{References}

Bavdekar, S. B. (2016). Formulating the right title for a research. Journal of the Association of Physicians of India, 64(2), 53-56. PMID: 27730781.

Beauchamp, T., \& Childress, J. (2001). Principles of Biomedical Ethics (5th ed.). Oxford, UK: Oxford University Press.

Bernard, H. R. (2002). Research methods in anthropology: Qualitative and quantitative approaches (3rd ed.). Walnut Creek, CA: Altamira Press.

Borbasi, S., \& Jackson, D. (2012). Navigating the Maze of Research. Chatswood, Sydney: Mosby Elsevier.

Burns, N., \& Grove, S. (2001). The Practice of Nursing Research: Conduct, Critique and Utilisation. WB Saunders: Philadelphia, USA.

Charmaz, K. (2014). Constructing grounded theory. London, United Kingdom: SAGE.

Connell, M. T. (1999). The research critique. In Tracy P. H. (Eds.), Nursing Research and Design (p. 57-74). UCD Press: Dublin.

Creswell, J., \& Plano-Clark, V. (2011). Designing and Conducting Mixed Methods Research (2nd ed.). Sage Publications: Los Angeles, USA.

Creswell, J. W., \& Zhang, W. (2009). The application of mixed methods designs to trauma research. Journal of Traumatic Stress: Official publication of the international society for traumatic stress studies, 22(6), 612-621. https://doi.org/10.1002/jts.20479

Denzin, N., \& Lincoln, Y. (2011). Qualitative research. Sage Publications: Thousand Oaks, USA.

Drisko, J. (2005). Writing up qualitative research. Families in Society. The Jour $\neg$ nal of Contemporary Social Services, 86(4), 589-593. https://doi.org/10.1606/1044-3894.3465

Flick, U. (2014). The Sage Handbook of Qualitative Data Analysis. Sage: London, UK.

Fossey, E., Harvey, C., Mcdermott, F., \& Davidson, L. (2002). Understanding and Evaluating Qualitative Research. Australian \& New Zealand Journal of Psychiatry, 36(6), 717-732. https://doi.org/10.1046/j.1440-1614.2002.01100.x.

Fowler, M. D. M. (2010). Guide to the Code of Ethics for Nurses: Interpretation and Application. Silver Spring, MD: American Nurses Association.

Frey, J., \& Fontana, A. (1993). The group interview in social research. In D. L. Morgan (Ed.), Successful Focus Groups, Advancing the State of the Art. Sage Publications: Newbury Park.

Gantley, M., Harding, G., Kumar, S., \& Tissier, J. (1999) Introduction to qualitative methods for health professionals. In Y., Carter, S., Shaw, \& C., Thomas, (Eds.), Master Classes in Primary Care Research Series. Royal College of General Practitioners: London.

Glaser, B., \& Strauss, A. L. (1967). The Discovery of Grounded Theory: Strategies for Qualitative Research. Chicago: Aldine. https://doi.org/10.1097/00006199-196807000-00014

Guba, E. G., \& Lincoln, Y. S. (1989). Fourth generation evaluation. Newbury Park, CA: Sage.

Hallberg L. R. (2010). Some thoughts about the literature review in grounded theory studies. International journal 
of qualitative studies on health and well-being, 5(3). https://doi.org/10.3402/qhw.v5i3.5387

Hemingway, S., Atkin, L., \& Stephenson, J. (2013). Assessing and managing wounds in mental health settings. Wounds UK, 9(3), 34-40.

Hinchliffe, M., \& Linthwaite, A. (2019). The use of LQD® spray dressing in patients with acute and chronic wounds in two mental health settings. Wound Care Today, 1(1), p 1-12.

Houser, J. (2018). Nursing research: Reading, using, and creating evidence. Jones \& Bartlett Learning: Sudbury, USA.

Issari, P., \& Pourkos, M. (2015). Qualitative research methods in Psychology and Education Kallipos [E-book]. Athens: Hellenic Academic books. Retrieved from https://hdl.handle. net/11419/ 5826.

Kedraka, K. (2008). Methodology for a successful interview (in Greek) [Online]. Retrieved from www.adulteduc.gr

King, N., \& Horrocks, C. (2010). Interviews in qualitative research. Sage Publications: London, UK.

Krueger, R. (1988). Focus groups: A practical guide for applied research. Sage Publications: USA.

Mantzoukas, S. (2007). Qualitative research in six easy steps: the epistemology, the methods and the presentation. Nursing, 46, 88-98.

Mantzoukas, S. (2004) Issues of representation within qualitative inquiry. Qualitative Health Research, 1144, 994-1007. https://doi.org/10.1177/1049732304265959

Mason, J. (2018). Qualitative researching (3d ed.). Sage Publications: London, UK.

Morris, D., \& Ecclesfield, N. (2011). A new computer-aided technique for qualitative document analysis. International Journal of Research \& Method in Education, 34(3), p241-254. https://doi.org/10.1080/1743727X.2011.609547

Patton, M. Q. (1999). Enhancing the quality and credibility of qualitative analysis. Health services research, 34(5 Pt 2), 1189-1208.

Patton, M. Q. (2002). Qualitative research and evaluation methods (3rd ed.). Thousand Oaks, CA: Sage Publications.

Pegram, A., \& Bloomfield, J. (2010). Wound care: principles of aseptic technique. Mental Health Practice, 14(2), 14-18. https://doi.org/10.7748/mhp2010.10.14.2.14.c8008

Polit, D. F., \& Beck, C. T. (2008). Nursing research: Generating and assessing evidence for nursing practice. Philadelphia: Wolters Kluwer Health/Lippincott Williams \& Wilkins.

Polit, E., \& Beck, T. (2006). Essentials of Nursing Research (6th ed.). Lippincott Williams \& Wilkins, Philadelphia.

Punch, K. (2005). Introduction to Social Research-Quantitative \& Qualitative Approaches. Sage Publications: London, UK, 2005.

Rice, P., \& Ezzy, D. (1999). Qualitative Research Methods: A Health Focus. Oxford University Press: South Melbourne, Australia.

Robson, C. (2010). Real World Research (K. Michalopoulou, Ed., V. P. Dalakou \& K. Vasilikou, Trans.). Athens, Greece: Gutenberg. (Original work published 1993) [In Greek]

Ryan, F., Coughlan, M., \& Cronin, P., (2007). Step-by-step guide to critiquing research. Part 2: qualitative research. British Journal of Nursing, 16(12), 738-744. https://doi.org/10.12968/bjon.2007.16.12.23726

Sanjari, M., Bahramnezhad, F., Fomani, F. K., Shoghi, M., \& Cheraghi, M. A. (2014). Ethical challenges of researchers in qualitative studies: The necessity to develop a specific guideline. Journal of medical ethics and history of medicine, 7. PMID: 25512833; PMCID: PMC4263394.

Stenius, K., Mäkelä, K., Miovský, M., \& Gabrhelík, R. (2017). How to Write Publishable Qualitative Research. In T. F., Babor, K., Stenius, R., Pates, M., Miovský, J., O'Reilly, \& P., Candon (Eds.), Publishing Addiction Science: A Guide for the Perplexed (pp. 155-172). London: Ubiquity Press. https://doi.org/10.5334/bbd.h

Vasileiou, K., Barnett, J., Thorpe, S., \& Young, T. (2018). Characterising and justifying sample size sufficiency in interview-based studies: systematic analysis of qualitative health research over a 15 -year period. $B M C$ Medical Research Methodology, 18, 148. https://doi.org/10.1186/s12874-018-0594-7 
Vaughn, P., \& Turner, C. (2016). Decoding via Coding: Analyzing Qualitative Text Data Through Thematic Coding and Survey Methodologies. Journal of Library Administration, 56(1), 41-51. https://doi.org/10.1080/01930826.2015.1105035

Vivar, C., McQueen, A., Whyte, D., \& Armayor, N. (2007). Getting started with qualitative research: developing a research proposal. Nurse researcher, 14(3), 60-73. https://doi.org/10.7748/nr2007.04.14.3.60.c6033

Wilkinson, S. (1998). Focus group methodology: a review. International Journal of Social Research Methodology, 1(3), 181-203. https://doi.org/10.1080/13645579.1998.10846874

Willig, C. (2008). Introducing qualitative research in psychology: adventures in theory and method. McGraw-Hill Education: USA.

Yin, R. (2011). Qualitative Research from Start to Finish. New York: Guilford.

\section{Copyrights}

Copyright for this article is retained by the author(s), with first publication rights granted to the journal.

This is an open-access article distributed under the terms and conditions of the Creative Commons Attribution license (http://creativecommons.org/licenses/by/4.0/). 